\title{
Influencia del Sistema de Calidad e Innovación Tecnológica en los Resultados Enfocados en la Mejora Continua en la manufactura de Transformadores de Distribución y Potencia
}

RECIBIDO:26/10/2017 ACEPTADO: 21/05/2018

Jorge CARMelo Ramos CARrióN ${ }^{1}$

\begin{abstract}
RESUMEN
El presente artículo expone los resultados de una investigación aplicada de tipo explicativa, que tuvo como objetivo analizar y determinar la influencia de la gestión del sistema de calidad y de la innovación tecnológica en los resultados enfocados en la mejora continua, en la manufactura de transformadores de distribución y potencia. La muestra en estudio estuvo constituida por el personal de empresas fabricantes del sector, ubicadas en Lima Metropolitana. La metodología desarrollada logró determinar el nivel de impacto utilizando un modelo de regresión lineal múltiple.
\end{abstract}

Palabras-claves: Sistema de Calidad; Innovación Tecnológica; Resultados Enfocados en la Mejora Continua.

INFLUENCE OF THE QUALITY SySTEM AND TeCHNOLOgical InNOVATION IN THE RESULTS Focused on CONTINUOUS IMPROVEMENT IN THE MANUFACTURE OF DISTRIBUTION AND POWER TRANSFORMERS

\section{ABSTRACT}

This article presents the results of an applied research of an explanatory type, whose objective was to analyze and determine the influence of the management of the quality system and technological innovation in the results focused on the continuous improvement in the manufacture of transformers distribution and power. The sample in study was constituted by the personnel of companies manufacturers of the sector, located in Metropolitan Lima. The methodology developed was able to determine the level of impact using a multiple linear regression model.

Keywords: Quality System; Technological Innovation; Results Focused on the Continuous Improvement.

\section{INTRODUCCIÓN}

En la actualidad la gestión del sistema de calidad y de la innovación tecnológica se han convertido en actividades muy importantes que contribuyen con la competitividad y el posicionamiento de la industria peruana, que afronta el gran desafío de adecuarse al mundo globalizado. Es por esta razón, que los gestores de las empresas fabricantes de transformadores de distribución y potencia, en la búsqueda de nuevas formas para mejorar la calidad de sus procesos y productos, se compenetren en procesos de mejora continua y requieran conocer oportunamente el nivel de influencia o impacto que tienen sobre ellos el sistema de calidad y la innovación tecnológica.

En este contexto se formula el problema general ¿Cómo influye el sistema de calidad y la innovación tecnológica en los resultados enfocados en la mejora continua, en la manufactura de transformadores de distribución y potencia? Con los siguientes problemas específicos: ¿Cómo influye el sistema de calidad en los resultados enfocados en la mejora continua, en la manufactura de transformadores de distribución y potencia? y ¿Cómo influye la innovación tecnológica en los resultados enfocados en la mejora continua, en la manufactura de transformadores de distribución y potencia?

La investigación procura hacer una contribución al estudio de la gestión de calidad y mejora continua de los procesos, presentando una metodología para evaluar y medir el impacto de las prácticas de gestión - del sistema de calidad e innovación tecnológica - sobre los resultados enfocados en la mejora continua. La importancia radica en que permitirá a la organización tomar medidas y acciones correctivas eficaces oportunamente.

El objetivo general del estudio es determinar cómo influye el sistema de calidad y la innovación tecnológica en los resultados enfocados en la mejora continua, en la manufactura de transformadores de distribución y potencia. Siendo los objetivos específicos; a) Determinar cómo influye el sistema de calidad en los resultados enfocados en la mejora continua, en la manufactura de transformadores de distribución y potencia; b) Determinar cómo

1 Maestro en Ciencias con mención en Sistemas de Potencia.

Docente Facultad de Ingeniería Eléctrica y Electrónica, UNI. Perú.

E-mail: jorgeramosc@uni.edu.pe.

https://orcid.org/ 0000-0001-9281-106X 
influye la innovación tecnológica en los resultados enfocados en la mejora continua, en la manufactura de transformadores de distribución y potencia.

\section{MARCO TEÓRICO}

\subsection{Gestión de Calidad}

Esta filosofía se entiende como un enfoque que ayuda a las empresas a obtener mayores resultados. Entre algunas de sus principales ventajas se tiene: la satisfacción de las necesidades y expectativas del cliente, el desarrollo de procesos de mejora, el compromiso de la dirección, la identificación y gestión de los procesos clave y el desarrollo de una visión de calidad (Giménez, Jiménez y Martínez, 2014). El sistema de gestión de la calidad debe ser considerado como un sistema que fortalece al sistema de gestión de la empresa (Cruz, 2004). La mejora continua se puede definir como un proceso planificado, organizado y sistemático de cambio continuo, que está basado en el ciclo Deming, y que en general comprende cuatro fases: el estudio de la situación actual; implantar las propuestas más convenientes; comprobar si se obtienen los resultados esperados; e implantar y estandarizar las mejoras con las modificaciones que fueren necesarias. La implantación de la mejora debe ser extendida en toda la empresa y realizada por todo el personal de la organización, para que así pueda ser denominada mejora continua (García, 2009).

\subsection{Gestión de la Innovación}

Es el proceso orientado a organizar y dirigir todos los recursos con el propósito de incrementar la creación de conocimiento y generar ideas para obtener nuevos procesos y productos con éxito en los mercados (Gallego, 2005). La norma UNE (Una Norma Española) 166002 define los requisitos que debe cumplir un sistema de gestión de la investigación, desarrollo e innovación; y puesto que este sigue la estructura de la metodología del ciclo planificar, hacer, controlar y actuar, se puede integrar también con el sistemas de gestión de calidad (Santos, 2017).

\subsection{Factores Claves de Éxito}

Del análisis de las variables de influencia del proceso de manufactura de transformadores de distribución y potencia, se pueden identificar los siguientes factores claves de éxito: a) Sistema de Calidad, que garantiza el aseguramiento de la calidad de los procesos de manufactura para satisfacer de manera óptima las necesidades, requerimientos y expectativas del cliente. Las organizaciones que desean ser eficientes crean y utilizan sistemas de calidad
(Summers, 2006); b) Innovación Tecnológica, que establece mecanismos que fomentan el desarrollo y la aplicación de la innovación al producto, a los procesos de manufactura y a la gestión de la calidad, así como a la inversión en tecnología para la producción y el producto (Rubio y Aragón, 2002); c) Resultados Enfocados en la Mejora Continua, que permiten determinar si la gestión y administración de los recursos, la investigación y el desarrollo de nuevos diseños, procesos tecnológicos y productos con calidad, contribuyen con los resultados alcanzados en la satisfacción del cliente, satisfacción del personal, desempeño de los productos y eficacia de los procesos.

\subsection{Transformadores de Distribución y Potencia}

"Se denomina transformador a un aparato electromagnético estático destinado para transformar un sistema, primario, de corriente alterna en otro, secundario, con la misma frecuencia, pero con otras características, en particular, tensión y corriente distintas" (Kostenko y Pietrovski, 1975, p.385). Los transformadores por su operación (Ras, 1994) se pueden clasificar como transformadores de distribución (Gutiérrez, Panteleeva, Hurtado y Gonzales, 2013) y transformadores de potencia. Esta denominación se debe a que son utilizados para suministro eléctrico tanto en sistemas de distribución como en sistemas de potencia.

Los transformadores están conformados por la cuba, la parte activa, el aceite aislante y los accesorios de protección y conexión. La parte activa es el componente más importante e integrado por el núcleo magnético, los arrollamientos y el sistema de aislamiento. Los arrollamientos se identifican tomando en consideración el nivel de tensión de servicio de la red eléctrica a la cual se van a conectar, así se tiene: arrollamiento de alta tensión, arrollamiento de media tensión y arrollamiento de baja tensión. Por lo general, los transformadores de distribución se fabrican con un rango de potencia de hasta 2000 Kilovoltioamperios (KVA) y tensión máxima de servicio de hasta 36 Kilovoltios (kV); en tanto que los transformadores de potencia se fabrican con una potencia superior a 3000 Kilovoltioamperios (KVA) y tensión máxima de servicio que supera los 60 Kilovoltios (kV).

\section{METODOLOGÍA DE LA INVESTIGACIÓN}

El presente estudio se planteó como una investigación aplicada, de tipo explicativa y de diseño cuantitativo no experimental y transversal. 


\subsection{Hipótesis General.}

$\boldsymbol{H}_{1}$ : El sistema de calidad y la innovación tecnológica influyen en los resultados enfocados en la mejora continua, en la manufactura de transformadores de distribución y potencia.

\subsection{Hipótesis Específicas.}

$\boldsymbol{H}_{1.1}$ : El sistema de calidad influye en los resultados enfocados en la mejora continua, en la manufactura de transformadores de distribución y potencia. $\boldsymbol{H}_{1.2}$ : La innovación tecnológica influye en los resultados enfocados en la mejora continua, en la manufactura de transformadores de distribución y potencia.

\subsection{Variables de las Hipótesis}

Variables independientes: Sistema de Calidad, es el "Conjunto de la estructura de organización, de responsabilidades, de procedimientos, de procesos y de recursos, que se establecen para llevar a cabo la gestión de calidad" (Cuatrecasas, 2010, p.336). Innovación Tecnológica, es la introducción de cualquier mejora o novedad en las operaciones tecnológicas, o la introducción de un bien o servicio que es nuevo o significativamente mejorado con respecto a sus características o funciones anteriores (Camisón y Villar, 2010).

Variable dependiente: Resultados Enfocados en la Mejora Continua, se refiere a la medición del desempeño de los resultados de la mejora continua, relacionados tanto con la organización como con sus clientes. "Desde el punto de vista de la primera, los resultados son los objetivos que se desea lograr. Desde la perspectiva de la segunda, los resultados representan aquello que esperan obtener al entablar una relación comercial con la organización" (Summers, 2006, p.161).

\subsection{Unidad de Análisis y Tamaño de la Muestra}

La unidad de análisis de la investigación estuvo constituida por las prácticas de gestión de calidad y mejora continua del personal que laboraba en el área de producción de cuatro empresas en estudio, y que se desempeñaban como directivos, administrativos y técnicos. Dichas empresas pertenecen al sector industrial manufacturero de transformadores de distribución y potencia de Lima Metropolitana. Las razones que se tomaron en cuenta para estudiarlas fueron su antigüedad en la actividad de producción de transformadores de distribución y potencia y el estar inmersos en los sistemas de gestión de calidad.

Para determinar el tamaño de la muestra se consideró que las variables poblacionales eran cualitativas y de distribución finita, por ello se calculó el total de personal a encuestar aplicando la siguiente fórmula.

$$
\boldsymbol{n}=\frac{\boldsymbol{N} * \boldsymbol{Z}^{2} * \boldsymbol{p} * \boldsymbol{q}}{(\boldsymbol{N}-\mathbf{1}) \boldsymbol{E}^{2}+\boldsymbol{Z}^{2} * \boldsymbol{p} * \boldsymbol{q}}
$$

Siendo, $\boldsymbol{N}$ : Tamaño de la población; $\boldsymbol{n}$ : Tamaño de la muestra; $\boldsymbol{E}$ : Margen de error estimado; $\boldsymbol{Z}$ : Distribución estándar de acuerdo al nivel de significancia; $\boldsymbol{p}$ : Proporción de la variable positiva; $\boldsymbol{q}$ : Proporción de la variable negativa $(1-p)$. Considerando que el tamaño de población era de 145 trabajadores y que no se conocía estadísticamente la proporción de la variable positiva, se asumió que $\boldsymbol{p}=\boldsymbol{q}=0.5$. Para un nivel de confianza del $95 \%$, de tablas de distribución de probabilidad normal (Quezada, 2010), $\boldsymbol{Z}=$ 1.96. De la fórmula 1 , se obtuvo que el tamaño de la muestra fuera de 105 trabajadores pertenecientes a las cuatro empresas industriales.

\subsection{Instrumento de Medición}

El instrumento de medición estuvo constituido por tres bloques que correspondían a los criterios (variables) en estudio con tres indicadores y nueve ítems por criterio, ver Tabla 1. En cada ítem se utilizó una escala ordinal discreta - con puntaje individual del uno al cinco en la escala de Likert - en base a cinco opciones posibles: (1) Totalmente en desacuerdo, (2)

Tabla 1. Variables e Indicadores del Instrumento de Medición

\begin{tabular}{|l|l|c|}
\hline Criterios (Variables) & Indicador & ítems \\
\hline \multirow{3}{*}{ 1 Sistema de calidad } & 1.1 dentificación y aplicación de los procesos & 3 \\
\cline { 2 - 3 } & 1.2 Documentación y control de los procesos & 3 \\
\cline { 2 - 3 } & 1.3 Seguimiento y mejora de la calidad & 3 \\
\hline \multirow{3}{*}{2 Innovación tecnológica } & 2.1 Actividades de innovación & 3 \\
\cline { 2 - 3 } & 2.2 Innovación del producto & 3 \\
\cline { 2 - 3 } 3 Resultados enfocados en la mejora continua & 2.3 Innovación de los procesos & 3 \\
\cline { 2 - 3 } & 3.1 Resultados enfocados al cliente & 3 \\
\cline { 2 - 3 } & 3.2 Resultados enfocados a los procesos y producto & 3 \\
\hline
\end{tabular}

Fuente: Elaboración propia 
En desacuerdo, (3) Ni de acuerdo ni en desacuerdo, (4) De acuerdo y (5) Totalmente de acuerdo; los valores entre paréntesis fueron los puntajes individuales.

\subsection{Análisis e Interpretación aplicando un Mode- lo de Regresión Lineal Múltiple}

Para explicar las relaciones e influencia que existían entre el sistema de calidad e innovación tecnológica sobre los resultados enfocados en la mejora continua, se utilizó un modelo de Regresión Lineal Múltiple. Con este modelo se respondió a la pregunta de investigación ¿Cómo influye el sistema de calidad y la innovación tecnológica en los resultados enfocados en la mejora continua, en la manufactura de transformadores de distribución y potencia? La determinación de este impacto permitió contrastar las hipótesis planteadas, a partir de la siguiente ecuación del modelo de regresión:

$$
R E M C_{i}=\beta_{01}+\beta_{1} S C_{i}+\beta_{2} I T_{i}+\varepsilon_{1 i}
$$

En donde, $\boldsymbol{R} \boldsymbol{E} \boldsymbol{M C}=$ Resultados Enfocados en la Mejora Continua; $\boldsymbol{S C}=$ Sistema de Calidad; $\boldsymbol{I T}=$ Innovación Tecnológica. En la ecuación del modelo de regresión, $i$ representa el número de observación para cada variable y asume los valores $i=1,2,3, \ldots, 105$. Siendo estas observaciones las respuestas a las encuestas que dieron cada uno de los 105 trabajadores, de las diferentes empresas en estudio. La constante $\boldsymbol{\beta}_{\mathbf{0 1}}$ representa al término independiente de la ecuación de regresión. Las constantes que representan a los coeficientes de regresión de las variables independientes fueron $\boldsymbol{\beta}_{\mathbf{1}}$ y $\boldsymbol{\beta}_{\mathbf{2}}$ . La constante $\varepsilon_{1 i}$ representa el término de error del modelo de regresión para la observación $i$.

En la interpretación de los resultados del modelo de regresión se consideraron las siguientes pruebas (Montgomery, 2008): a) Prueba de significancia de la regresión; b) Prueba de los coeficientes de regresión individuales, que proporciona un valor del coeficiente estandarizado $\boldsymbol{\beta}$ que mide el impacto que tiene la variable predictor sobre la variable respuesta (dependiente), según la Tabla 2; c) Bondad de ajuste.

Tabla 2. Coeficientes de Regresión e Impactos

\begin{tabular}{|c|l|}
\hline Coeficiente de Regresión & \multicolumn{1}{|c|}{ Valoración } \\
\hline Menor a 0.10 & Impacto Imperceptible \\
\hline De 0.11 a 0.15 & Impacto Apenas Perceptible \\
\hline De 0.16 a 0.19 & Impacto a Considerar \\
\hline De 0.20 a 0.29 & Impacto Importante \\
\hline De 0.30 a 0.50 & Impacto Fuerte \\
\hline Mayores a 0.50 & Impacto muy Fuerte \\
\hline
\end{tabular}

Fuente. Elaboración propia en base a Rositas (2005), p.140.

\section{ANÁLISIS E INTERPRETACIÓN DE RESUL- TADOS}

Para la identificación y determinación de las variables de influencia de los modelos de regresión lineal múltiple, se aplicó el método de regresión Paso a Paso del software SPSS Statistics 20. Este método determina la incorporación automática de las variables predictores al modelo de regresión, siempre que estas cumplieran los siguientes criterios: nivel de significancia $\leq 0.05$ para introducir a la variable y nivel de significancia $\geq 0.10$ para excluir a la variable.

Para evaluar la calidad de los datos obtenidos en la muestra de la población, se verificó el cumplimiento de la fiabilidad y validez del instrumento de medición, así como el cumplimiento de los supuestos de normalidad y homogeneidad de las variables intervinientes en el modelo.

\subsection{Fiabilidad, Validez, Normalidad y Homoge- neidad}

En las empresas en estudio se aplicó el instrumento de medición a un total de 105 trabajadores de la línea de producción de transformadores de distribución y potencia, clasificados de acuerdo a las funciones que desempeñaban en tres categorías, correspondiéndoles los siguientes totales: 21 trabajadores como jefes o supervisores, 28 trabajadores como personal administrativo y 56 trabajadores como personal técnico u operario.

En la Tabla 3, se presentan los resultados de los supuestos de fiabilidad y validez del instrumento que midió los niveles de práctica de gestión, tanto del sistema de calidad como de innovación tecnológica, así como los resultados enfocados en la mejora continua.

En la prueba de Fiabilidad, el Alfa de Cronbach obtuvo un estadístico con significancia mayor a 0.70 (Méndez y Gómez, 2017), por consiguiente el instrumento de medición cumplía con el supuesto de fiabilidad. De la prueba de adecuación muestral Kaiser, Meyer y Olkin (KMO), se obtuvo un estadístico con significancia mayor a 0.80 (Frías y Pascual, 2012), determinándose que las variables del modelo estaban bien correlacionadas en el instrumento de medición. De la realización del test de esfericidad de Bartlett, se obtuvo un estadístico con significancia $p<0.05$, rechazándose la hipótesis nula con el $95 \%$ de confianza (Pérez, 2009), por tanto existía una correlación significativa entre las variables del instrumento de medición. En la prueba de Comunalidad, para todas las variables, se obtuvieron estadístico con significancia mayor a 0.50 (Frías y Pascual, 2012), lo que indicaba que los 
factores explicaban la variabilidad de la variable dependiente. También se comprobó el cumplimiento de los supuestos estadísticos subyacentes a las variables que intervenían en los modelos de regresión, como son la normalidad (Kolmogorov-Smirnov) y la homogeneidad de la varianza (Levene). Los resultados se muestran en el Tabla 4.

De la realización de estas pruebas, para todas las variables del modelo, se obtuvieron estadísticos con nivel de significancia $p>0.05$, aceptándose la hipótesis nula con el $95 \%$. Por consiguiente las variables del modelo cumplían con los supuestos de Normalidad y Homogeneidad de la Varianza (Homocedasticidad).

\subsection{Impacto en los Resultados Enfocados en la Mejora Continua}

Del análisis de regresión se obtuvieron los valores de las correlaciones entre las variables indepen- dientes y dependientes del modelo de regresión que se presentan en el Tabla 5.

En esta tabla, se puede apreciar que los Resultados Enfocados en la Mejora Continua tienen alta correlación con las variables independientes Sistema de Calidad $(0.708 ; p<0.001)$ e Innovación Tecnológica (0.647; $p<0.001)$. También se puede comprobar que dichas variables independientes no tienen una fuerte correlación entre ellas (valor cercano a uno), lo que determina que existe independencia entre estas variables.

En la Tabla 6, se presentan los valores de la Bondad de Ajuste del Modelo de Regresión que mejor explicaba a la variable dependiente Resultados Enfocados en la Mejora Continua.

Este modelo de regresión explica, en porcentajes, el $53.70 \%$ de la variable dependiente Resultados

Tabla 3. Estadísticos de Fiabilidad y Validez

\begin{tabular}{|c|c|c|c|}
\hline FIABILIDAD & \multicolumn{3}{|c|}{ VALIDEZ } \\
\hline Alfa de Cronbach & Prueba KMO & Test de Bartlett $\boldsymbol{X}^{\mathbf{2}}$ & Comunalidad \\
\hline 0.972 & 0.905 & $588.922^{* * *}$ & Mayor a 0.617 \\
\hline N: 105 Trabajadores; 27 Elementos; ${ }^{* * *}$ Significación $<0.001$ (Altamente significativo) \\
\hline
\end{tabular}

Fuente. Elaboración propia.

Tabla 4. Pruebas de Normalidad y Homogeneidad de la Varianza

\begin{tabular}{|l|l|l|l|l|}
\hline \multirow{2}{*}{ Variables } & \multicolumn{2}{c|}{ Kolmogorov-Smirnov } & \multicolumn{2}{c|}{ Levene } \\
\cline { 2 - 5 } & \multicolumn{1}{c|}{ Estadístico } & \multicolumn{1}{c|}{ Sig. } & Estadístico $\left.{ }^{* *}\right)$ & Sig. \\
\hline Sistema de Calidad & 0.983 & 0.289 & 1.620 & 0.152 \\
\hline Innovación Tecnológica & 1.334 & 0.057 & 2.355 & 0.100 \\
\hline Resultados Enfocados en la Mejora Continua & 1.281 & 0.075 & 2.311 & 0.104 \\
\hline${ }^{* *}$ Con 2 y 102 Grados de libertad & \multicolumn{5}{|l}{} \\
\hline
\end{tabular}

Fuente. Elaboración propia.

Tabla 5. Correlaciones de las Variables

\begin{tabular}{|l|c|c|c|}
\hline Correlación de Pearson & $\begin{array}{c}\text { Resultados Enfocados } \\
\text { en la Mejora Continua }\end{array}$ & Sistema de Calidad & Innovación Tecnológica \\
\hline Resultados Enfocados en la Mejora Continua & 1.000 & $0.708^{* * *}$ & $0.647^{* * *}$ \\
\hline Sistema de Calidad & $0.708^{* * *}$ & 1.000 & $0.729^{* * *}$ \\
\hline Innovación Tecnológica & $0.647^{* * *}$ & $0.729^{* * *}$ & 1.000 \\
\hline N: Total de trabajadores & 105 & 105 & 105 \\
\hline$\left(^{* * *}\right)$ Nivel de Significancia $<0.001$ & & \\
\hline
\end{tabular}

Fuente. Elaboración propia.

Tabla 6. Bondad de ajuste del Modelo de Regresión

\begin{tabular}{|c|c|c|c|}
\hline $\mathbf{R}$ & $\mathbf{R}$ cuadrado & R cuadrado corregida & Durbin Watson \\
\hline $0.733^{\text {a }}$ & 0.537 & 0.528 & 1.764 \\
\hline a. Variables predictores: Constante, Sistema de Calidad, Innovación Tecnológica \\
\hline
\end{tabular}

Fuente. Elaboración propia. 
Enfocados en la Mejora Continua, que una vez corregido por el efecto de la muestra y de las variables independientes resulta ser de $52.80 \%$. Por otro lado, el estadístico de Durwin - Watson resulta ser de 1.764 (No existe auto correlación entre los residuos).

La prueba de significancia del modelo se realizó a través del Análisis de la Varianza, cuyos resultados se presentan en la Tabla 7 . En ella se puede apreciar que el valor del estadístico $\boldsymbol{F}$ fue de 59.218 con una significancia de 0.000 ; lo que llevó a rechazar la hipótesis nula con el $95 \%$ de confianza $(p<0.05)$ y considerar que existe un efecto real y lineal de las variables Sistema de Calidad e Innovación Tecnológica sobre la variable Resultados Enfocados en la Mejora Continua.

Los resultados de la prueba de significancia de los coeficientes de regresión individuales se presentan en el Tabla 8.

De estos resultados, Tabla 8, se deduce que las variables independientes Sistema de Calidad e Innovación Tecnológica contribuyen significativamente con los Resultados Enfocados en la Mejora Continua ( $p<0.05$ ), por tanto la ecuación de regresión del modelo con coeficientes no estandarizados puede representarse como:

$$
\widehat{R E M C}=1.705+0.394 S C+0.181 I T
$$

Siendo, $\widehat{\boldsymbol{R E M} C}=$ Estimación de los Resultados Enfocados en la Mejora Continua; $\boldsymbol{S C}=$ Sistema de Calidad; $\boldsymbol{I T}=$ Innovación Tecnológica .
En el modelo se puede apreciar que por cada unidad de valor medio que se incremente el Sistema de Calidad, manteniéndose constante el valor medio de la Innovación Tecnológica, los Resultados Enfocados en la Mejora Continua se incrementarán en 0.394 unidades de valor medio. De igual forma, por cada unidad de valor medio que se incremente la Innovación Tecnológica, manteniéndose constante el valor medio del Sistema de Calidad, los Resultados Enfocados en la Mejora Continua se incrementarán en 0.181 unidades de valor medio. Para determinar la importancia o impacto del predictor sobre los Resultados Enfocados en la Mejora Continua, expresamos la ecuación de regresińn del modelo con los coeficientes estandarizados $\boldsymbol{\beta}$, obteniéndose:

$$
\widehat{R E M C}=0.504 S C+0.279 I T(4)
$$

Se observa que el impacto del Sistema de Calidad es superior al de la Innovación Tecnológica.

\subsection{Contrastación de Hipótesis}

La contrastación de la hipótesis general se realizó a través de la contrastación de las dos hipótesis específicas formuladas en la investigación; utilizando los resultados de las pruebas de significancia de los coeficientes no estandarizados del modelo de regresión lineal múltiple.

Hipótesis específica de la variable de influencia Sistema de Calidad

De la prueba de significancia de los coeficientes no estandarizados del modelo de regresión, Tabla

\begin{tabular}{|c|c|c|c|c|c|}
\hline Modelo & Suma de cuadrados & Grados de libertad & Media cuadrática & Estadístico F & Nivel de Significancia \\
\hline Regresión & 10.139 & 2 & 5.069 & 59.218 & \multirow{3}{*}{0.000} \\
\hline Residual & 8.732 & 102 & 0.086 & & \\
\hline Total & 18.871 & 104 & & & \\
\hline
\end{tabular}

Tabla 7. Análisis de Varianza del Modelo de Regresión

Fuente. Elaboración propia.

\begin{tabular}{|c|c|c|c|c|c|c|}
\hline \multirow{2}{*}{ Variables del Modelo } & \multirow{2}{*}{$\begin{array}{c}\text { Coeficientes no } \\
\text { estandarizados } \\
\text { B }\end{array}$} & \multirow{2}{*}{$\begin{array}{c}\text { Coeficientes } \\
\text { estandarizados } \\
\beta\end{array}$} & \multirow{2}{*}{$\begin{array}{c}\text { Estadístico } \\
\mathbf{t}\end{array}$} & \multirow{2}{*}{ Nivel de sig. } & \multicolumn{2}{|c|}{ Intervalo de confianza al $95 \%$} \\
\hline & & & & & Límite inferior & Límite superior \\
\hline Constante & 1.705 & & 8.025 & $0.000^{* *}$ & 1.284 & 2.127 \\
\hline Sistema de Calidad & 0.394 & 0.504 & 5.126 & $0.000^{* *}$ & 0.241 & 0.546 \\
\hline Innovación Tecnológica & 0.181 & 0.279 & 2.832 & $0.006^{*}$ & 0.054 & 0.309 \\
\hline
\end{tabular}

Tabla 8. Prueba de Significancia de los Coeficientes de Regresión

Fuente. Elaboración propia. 
8, se infiere lo siguiente: La variable predictor Sistema de Calidad obtuvo un estadístico $\boldsymbol{t}$ de valor 5.126, como el nivel de significancia fue de 0.000 se rechazó la hipótesis nula con el $95 \%$ de confianza $(p<0.05)$. Por consiguiente, el modelo de regresión lineal múltiple determinó que se acepta la hipótesis de investigación:

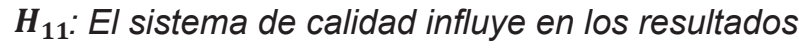 enfocados en la mejora continua, en la manu- factura de transformadores de distribución $y$ potencia.}

\section{b) Hipótesis específica de la variable de influen- cia Innovación Tecnológica}

De la prueba de significancia de los coeficientes no estandarizados del modelo de regresión, Tabla 8, se infiere lo siguiente: La variable predictor Innovación Tecnológica obtuvo un estadístico $\boldsymbol{t}$ de valor 2.832; como el nivel de significancia fue de 0.006 se rechazó la hipótesis nula con el $95 \%$ de confianza $(p<0.05)$. Por tanto, el modelo de regresión lineal múltiple determinó que se acepta la hipótesis de investigación:

$\boldsymbol{H}_{12}$ : La innovación tecnológica influye en los resultados enfocados en la mejora continua, en la manufactura de transformadores de distribución y potencia.

\section{c) Contrastación de la Hipótesis General ${ }_{1}$}

De la contrastación de las dos hipótesis específicas podemos inferir finalmente que se acepta la hipótesis general:
$\boldsymbol{H}_{1}$ : El sistema de calidad y la innovación tecnológica influyen en los resultados enfocados en la mejora continua, en la manufactura de transformadores de distribución y potencia.

\subsection{Interpretación de Resultados}

En la Figura 1, se aprecia el grado de impacto de cada variable independiente sobre la variable dependiente. Este grado de impacto se representa por el valor del coeficiente de regresión tipificado (estandarizado) ubicado en un rectángulo en cada flecha que describe la relación (entre las variables). En el mismo rectángulo y a un lado de cada coeficiente, se anota a qué nivel es estadísticamente significativa la relación explicada por el coeficiente; así tenemos, con un asterisco $\left(^{*}\right)$ si la relación es significativa al 0.05 y con dos asteriscos $\left({ }^{* *}\right)$ si la relación es significativa al 0.01 . A la variable que recibe impactos se le acompaña con el valor del coeficiente de determinación $R^{2}$. Para determinar el grado de impacto de la variable independiente sobre la variable dependiente, en la población de la muestra de empresas manufactureras de transformadores de distribución y potencia, utilizaremos la valoración de los Coeficientes de Regresión e Impactos que se presentaron en la Tabla 2.

El impacto del Sistema de Calidad en los Resultados Enfocados en la Mejora Continua es valorado como impacto positivo Muy Fuerte $\left(0.504^{* *}\right.$, $\left.R^{2}: 53.7 \%\right)$; con significancia estadística y aceptable coeficiente de determinación $R^{2}$, por tanto existe evidencia para determinar estadísticamente que se gestiona adecuadamente el aseguramiento de la

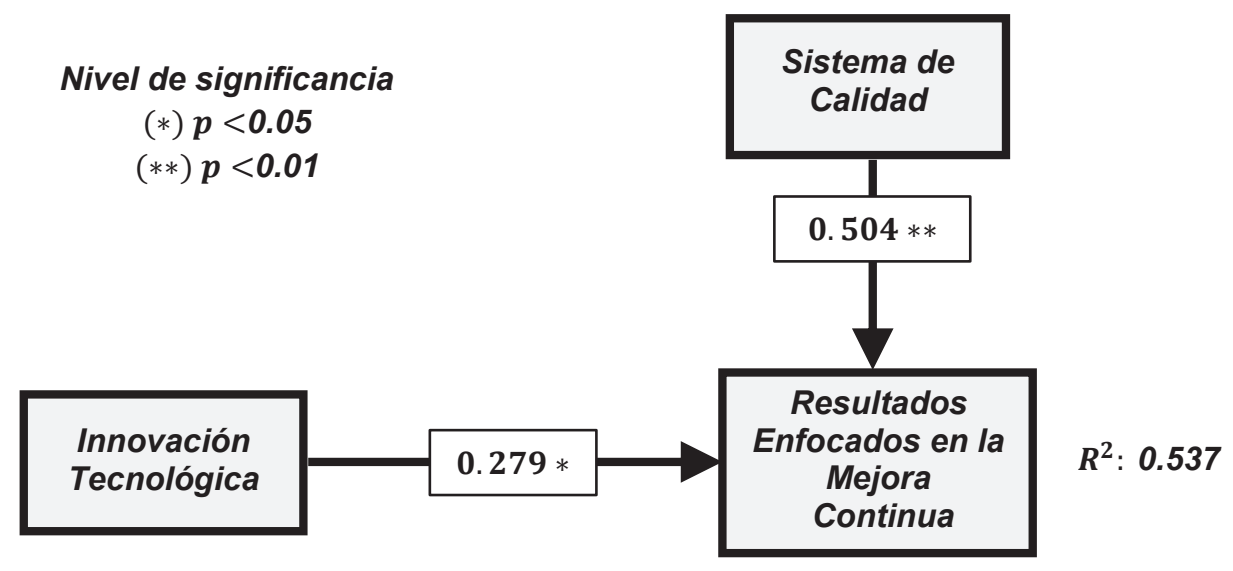

Figura 1. Modelo Gráfico Integral de los Resultados Enfocados en la Mejora Continua.

Fuente. Elaboración propia. 
calidad y que se impulsan e implementan acciones para garantizar la calidad del personal y de los proveedores, contribuyendo con los resultados alcanzados en la satisfacción del cliente, satisfacción del personal, desempeño de los productos y eficacia de los procesos, en la manufactura de los transformadores de distribución y potencia de la muestra en estudio.

El impacto de la Innovación Tecnológica en los Resultados Enfocados en la Mejora Continua es valorado como impacto positivo Importante $\left(0.279^{*}, R^{2}: 53.7 \%\right)$. La interacción tiene significancia estadíctica y aceptable coeficiente de determinación $R^{2}$, por tanto existe evidencia para determinar estadísticamente, que se gestiona una política de fomento de la innovación y desarrollo tecnológico así como de la implementación de ideas innovadoras; contribuyendo con los resultados alcanzados en la satisfacción del cliente, satisfacción del personal, desempeño de los productos y eficacia de los procesos, en la manufactura de los transformadores de distribución y potencia de la muestra en estudio.

\section{CONCLUSIONES}

Se probaron la hipótesis general y las hipótesis específicas formuladas en la investigación.

Se determinó el impacto que tienen el sistema de calidad y la innovación tecnológica en los resultados enfocados en la mejora continua, en la manufactura de transformadores de distribución y potencia de la muestra en estudio.

Se puede afirmar que el sistema de calidad tiene un impacto positivo Muy Fuerte en los resultados enfocados en la mejora continua, en la manufactura de transformadores de distribución y potencia de la muestra en estudio.

Se puede afirmar que la innovación tecnológica tiene un impacto positivo Importante en los resultados enfocados en la mejora continua, en la manufactura de transformadores de distribución y potencia de la muestra en estudio.

Los resultados revelaron que el personal directivo, administrativo y técnico está inmerso en las prácticas de gestión de calidad y mejora continua, en las empresas manufactureras de la muestra en estudio.

\section{REFERENCIAS BIBLIOGRÁFICAS}

Camisón, C. y Villar, A. (2010). Análisis del papel mediador de las capacidades de innovación tecnológica en la relación entre la forma organizativa flexible y el desempeño organizativo. Cuadernos de Economía y Dirección de la Empresa, 23(45), 115-144. $\quad$ https://doi.org/10.1016/S11385758(10)70026-8

Cuatrecasas, LL. (2010). Gestión integral de la calidad: Implantación, control y certificación. España: Editorial Profit.

Cruz, V. (2004). Sistema de Gestión de la Calidad en el Apoyo a la Implementación de Estrategias de Producción Ajustada. Información tecnológica, 15(6), 63-70. https://dx.doi.org/10.4067/S071807642004000600010

Frías, D. y Pascual, N. (2012). Prácticas del Análisis Factorial Exploratorio (AFE) en la investigación sobre conducta del consumidor y marketing. Suma Psicológica. 19(1), 4758. Recuperado de http://www.scielo.org.co/ scielo.php?script=sci_arttext\&pid=S012143812012000100004\&Ing=en\&tIng=es

Gallego, J. (2005). Fundamentos de la Gestión Tecnológica e Innovación. Revista Tecno Lógicas. (15), 113-131. Recuperado de https://dialnet.unirioja.es/servlet/ articulo? codigo $=506296$

García J. (2009). Barreras y facilitadores para la implantación, evolución y sostenibilidad de la mejora continua en proveedores del sector del automóvil. (Tesis Doctoral). Universidad Politécnica de Valencia. España. http://hdl. handle.net/10251/6222

Giménez, J., Jiménez, D. y Martínez, M. (2014). La gestión de calidad: importancia de la cultura organizativa para el desarrollo de variables intangibles. Revista Europea de Dirección y Economía de la Empresa. 23, 115-126. http:// dx.doi.org/10.1016/j.redee.2014.02.002

Gutiérrez, E., Panteleeva, O., Hurtado M. y González C. (2013). Aplicación de un modelo de inventario con revisión periódica para la fabricación de transformadores de distribución. Ingeniería Investigación y Tecnología, 14(4), 2013: 537-551. https://doi.org/10.1016/S14057743(13)72264-9 
Kostenko, M. y Piotrovski, L. (1975).Máquinas eléctricas: Tomo I. Moscú: Editorial MIR.

Méndez, M. y Gómez, M. (2017). Factores incidentes para crear valor compartido en las mipymes de Bogotá. Suma de Negocios. 8, (18), 96-105. https://doi.org/10.1016/j.sumneg.2017.10.003

Montgomery, D. (2008). Diseño y análisis de experimentos. México: Editorial Limusa Wiley.

Pérez, C. (2009). Técnicas de análisis de datos con SPSS 15. México: Editorial Pearson Prentice Hall.

Quezada, N. (2010). Metodología de la Investigación. Perú: Editorial MACRO.

Ras, E. (1994). Transformadores de potencia, de medida y de protección. España: Marcombo Editores.
Rositas, J. (2005). Factores críticos de éxito en la gestión de calidad y su grado de presencia e impacto en la industria manufacturera mexicana. (Tesis Doctoral). Universidad Autónoma de Nuevo León. México. http:// eprints.uanl.mx/id/eprint/1675

Rubio, A. y Aragón, A. (2002). Factores explicativos del éxito competitivo. Un estudio empírico en la pyme. Cuadernos de Gestión. 2(1), 49-63. https://addi.ehu.es/handle/10810/7024

Santos, D. (2017). Gestión de la Innovación en una PYME peruana de base tecnológica: un caso de estudio. (Tesis Maestría). Pontificia Universidad Católica del Perú. Perú. http://tesis.pucp.edu. pe/repositorio/handle/123456789/9983

Summers, D. (2006). Administración de la Calidad. México. Pearson-Educación. 
